\title{
Management Information Systems and Data Science in the Smart Grid - Inner Class Area Capacity Distribution of the iSHM Class Maps of Overhead Low-Voltage Broadband over Power Lines Topologies
}

\author{
Athanasios G. Lazaropoulos ${ }^{1,2, *}$ \\ 1: School of Electrical and Computer Engineering / National Technical University of Athens / \\ 9 Iroon Polytechniou Street / Zografou, GR 15780 \\ 2: Department of Industrial Design and Production Engineering / School of Engineering / University of \\ West Attica / 250 Thivon \& P. Ralli / Athens, GR 12244
}

Received August 20, 2020; Accepted September 21, 2020; Published September 27, 2020

\begin{abstract}
On the basis of the initial Statistical Hybrid Model (iSHM), the iSHM class maps, which are 2D contour plots and may graphically classify the real and virtual OV LV BPL topologies into five class areas, are upgraded in this paper by exploiting the third dimension of the capacity so that the upgraded class maps can provide additional information concerning the inner class area capacity distribution. The comprehension of the behavior of the inner class area capacity distribution is critical in order to deeper understand the extent and the position of iSHM class map footprints when various operation conditions of OV LV BPL topologies occur. Two inner class area capacity distribution rule of thumbs that deal with the OV LV BPL topology classification and capacity estimation are proposed thus supporting the management information system of OV LV BPL networks.
\end{abstract}

Keywords: Smart Grid; Broadband over Power Lines (BPL) networks; Power Line Communications (PLC); Distribution and Transmission Power Grids; Capacity, Statistics; Modeling; Management Information Systems; Data Science

\section{Introduction}

Broadband over Power Lines (BPL) technology can be considered to be one among the communications solutions, such as Radio Frequency (RF) mesh, modified Long Term Evolution (LTE), Code Division Multiple Access (CDMA) at sub GHz bands, dedicated fiber along high voltage lines and $5 \mathrm{G}$ communications, that is going to help towards the transformation of the existing power grid into an advanced IP-based communications network enhanced with a plethora of broadband smart grid applications [1], [2].

Since BPL technology exploits the available power grid infrastructure (i.e., Multiconductor Transmission Line (MTL) configurations and related power devices), which is anyway designed to deliver power rather to support communications, high and frequency-selective channel attenuation remains as one of the critical deficiencies of the 
BPL signal propagation and transmission. Among the different categories of BPL channel models, a great number of statistical BPL channel models, has been proposed in a variety of BPL technology application fields [3]-[14]. SHM that has been recently proposed in [7]-[9] is based on the Deterministic Hybrid Model (DHM), which has been exhaustively tested in transmission and distribution BPL networks [15]-[17], and is hereafter applied as the required SHM system input procedure. Actually, initial Statistical Hybrid Model (iSHM), which is one of the two supported versions of SHM and is employed in this paper, offers simulation results that are treated as the statistically processed DHM numerical results through a set of appropriate Channel Attenuation Statistical Distributions (CASDs); say, Gaussian, Lognormal, Wald, Weibull and Gumbel CASDs. Until now, the impact of a variety of parameters on the iSHM simulation results has been investigated so far such as the topology length, the interconnections between branches / main lines, branch lengths, distances between branches, branch terminations and channel attenuation measurement differences between the theoretical and practical results due to the real operation conditions [8], [18], [19]. Apart from the impact of the aforementioned intrinsic parameters, critical events during the operation of power grids, such as branch line faults and hook-style energy thefts, can be detected even if real operation conditions occur by exploiting the class maps footprints of iSHM [20], [21]; here, it should be reminded that a class map is a 2D contour plot that: (i) graphically classifies real and virtual BPL topologies in terms of their CASD Maximum Likelihood Estimator (MLE) parameter pairs and capacity; (ii) illustrates the borders between the BPL topology classes; and (iii) corresponds each CASD MLE parameter pair to its BPL topology subclass average capacity for given power grid type, CASD, coupling scheme, Injected Power Spectral Density Limits (IPSD) limits and noise Power Spectral Density (PSD) levels; while class map footprints are the graphical correspondence of CASD MLE parameter pair with the capacity that are represented on the class maps and may assess the impact of the intrinsic parameter change or the existence of critical events during the power grid operations. As the OV LV BPL topologies are examined in this paper, when changes of intrinsic parameters or the aforementioned critical events occur the respective CASD MLE parameters of the modified OV LV BPL topologies tend to change their iSHM footprint locations on the class maps following patterns of the same capacity behavior as presented in [20], [22]. In this paper, the inner class area capacity distribution of the iSHM class maps of OV LV BPL topologies is first investigated while the differences between the capacity of the aforementioned modified OV LV BPL topologies and the respective BPL topology subclass average capacities, which are used in class maps, for given CASD MLE parameter pairs is computed. On the basis of the findings of the inner class area capacity distribution of the iSHM class maps of OV LV BPL topologies and by exploiting the data science, the management information system of the BPL networks is further enhanced with two newly presented rules of thumb that allow: (i) the capacity assessment and the classification of OV LV BPL topologies by studying their CASD MLEs parameter pairs; (ii) the stability of the power grid; and (iii) the surveillance and monitoring of the power grid in order to identify possible critical events.

The rest of this short paper, which may act as a companion paper of [18], [20], [22], [23], is organized as follows: Section 2 briefly presents the theory concerning the iSHM class maps and iSHM class map footprints of OV LV BPL topologies. In Section 3, the simulation results regarding the inner class area capacity distribution are demonstrated as well as and the capacity differences between the modified OV LV BPL 
topologies and the respective BPL topology subclass average capacities of iSHM class maps. The two rules of thumb concerning the monitoring and surveillance of the OV LV BPL networks are here presented.

\section{2. iSHM Class Maps and iSHM Class Map Footprints}

Prior to numerically investigate the inner class area capacity distribution in iSHM class maps, brief details concerning the definition of iSHM class maps, the iSHM class map footprints of OV LV BPL topologies and the need for this capacity distribution study of class maps are here given. Note that the values of the required operation parameters for the interconnection and the fine application of DHM, iSHM, the iSHM class maps and the iSHM class map footprints of OV LV BPL topologies are reported in [22], [23].

\section{1 iSHM Class Maps}

Already been mentioned, SHM consists of two versions; say, iSHM and mSHM. With reference to the BPMN diagrams of iSHM [7], [23], iSHM that is the SHM version of interest in this paper consists of six Phases. The input parameters of iSHM, which coincide with the ones of DHM, are the topological characteristics of the examined real indicative OV LV BPL topologies, the applied coupling schemes, IPSD limits and noise PSD levels while the output of iSHM is the capacity range of each OV LV BPL topology class for given CASD. Also, iSHM supports five CASDs with their corresponding MLEs (i.e., Gaussian, Lognormal, Wald, Weibull and Gumbel CASDs). According to [20], each iSHM CASD exhibits different performance depending on the input parameters but Weibull CASD performs the best performance among the available ones in terms of the performance capacity metrics of the absolute threshold of percentage change and the average absolute percentage change when OV LV BPL topologies are examined. Hence, the CASD approximation accuracy to the real capacity results of [20] mandates the use of Weibull CASD for the further analysis of OV LV BPL topologies in this paper, which is anyway reevaluated for its accuracy during the critical events assumed in this paper.

To enrich the five OV LV BPL topology classes, which are straightforward defined after the initial selection of the five respective real indicative OV LV BPL topologies of Table 1 of [23], with other OV LV BPL topologies, the iSHM definition procedure, which has been proposed in [24], statistically defines virtual indicative OV LV BPL topologies by using iSHM CASD MLE parameter pairs and inserts the virtual topologies to the existing five OV LV BPL topology classes. The flowchart of the iSHM definition procedure for OV LV BPL topologies is given in Fig. 3(a) of [23]. Class maps are the output of the iSHM definition procedure where: (i) each CASD MLE parameter pair is corresponded to its OV LV BPL topology subclass average capacity; (ii) CASD MLE parameter pairs can describe real and virtual OV LV BPL topology subclasses; (iii) OV LV BPL topology class areas may be illustrated with respect to the computed capacity borders between the OV LV BPL topology classes; and (iv) with respect to the capacity borders of OV LV BPL topology classes and the Weibull CASD capacity performance, OV LV BPL topology subclasses can be arranged on the class maps by exploiting their CASD MLE parameter pairs. 


\section{2 iSHM Footprints on Class Maps}

iSHM footprints that are added on the class maps as groups of white spots can graphically assess the impact of the intrinsic parameter changes or the existence of critical events during the power grid operation and can help towards the quick identification of the critical events for future actions by the management information system of the smart grid. The theoretical definition of iSHM footprints has been presented in [23] while a variety of applications of iSHM footprints has been presented so far, such as the iSHM footprints of the real OV LV BPL topologies, of the OV LV BPL topologies with a sole branch line fault and of the OV LV BPL topologies with a single hook for energy theft [20]. By studying iSHM footprints of OV LV BPL topologies, it is clear that the iSHM footprint extent, size, white spot positions and white spot group direction with respect to the real indicative OV LV BPL topologies may imply the intrinsic parameter change or the nature of the occurred critical events during the power grid operation.

Until now, Weibull CASD is applied during the preparation of iSHM footprints of OV LV BPL topologies due to the best performance among the available supported iSHM CASDs in terms of the performance capacity metrics of the absolute threshold of percentage change and average absolute percentage change when the indicative real OV LV BPL topologies have been assumed [20]. The goal of this paper and of the following Section is to assess the capacity estimation accuracy of iSHM footprints for given Weibull CASD MLEs that come from the OV LV BPL topologies that suffer from intrinsic parameter change or critical events, briefly denoted as modified OV LV BPL topologies, and, hence, justifies the white spot positions and directions of the respective iSHM footprints on the iSHM class maps. The aforementioned assessment can be accomplished either qualitatively by further investigating the inner class area capacity distribution of the iSHM class maps or quantitatively by comparing the capacity of the modified OV LV BPL topologies with the average capacity of the OV LV BPL topology subclass of iSHM class maps whose CASD MLE parameter pair is the same with the one of the examined modified OV LV BPL topology.

\section{Numerical Results and Discussion}

In this Section, numerical results that investigate the inner class area capacity distribution of the iSHM class maps of OV LV BPL topologies are first demonstrated so that a clearer image of the relation between Weibull CASD MLE parameter pair and the OV LV BPL topology subclass average capacity can be shown. This study allows the easier matching between the Weibull CASD MLEs of the examined modified OV LV BPL topologies and the capacity as defined by the iSHM class maps. Then, for the cases of: (i) arbitrary real OV LV BPL topologies; (ii) real indicative OV LV BPL topologies with arbitrary branch line faults; and (iii) real indicative OV LV BPL topologies with arbitrary hook-style energy thefts, their capacities are reported and are compared against the average capacity of the OV LV BPL topology subclass of iSHM class maps whose CASD MLE parameter pair is the same with the one of the examined modified OV LV BPL topology. 


\section{1 iSHM Class Maps of OV LV BPL Topologies}

Already been mentioned, iSHM class maps of Weibull CASD are the basis where iSHM footprints of Weibull CASD are applied onto when the modified OV LV BPL topologies are going to be examined by the management information system of the smart grid. In accordance with [20], the iSHM class map of OV LV BPL topologies is plotted in Fig. 1(a) with respect to $\hat{a}_{\mathrm{MLE}}^{\mathrm{Weibull}}$ and $\hat{\beta}_{\mathrm{MLE}}^{\mathrm{Weibull}}$ for the default operation settings of [23] when the average capacity of each OV LV BPL topology subclass is considered. Note that as the spacings of the horizontal and vertical axes are concerned, they are assumed to be equal to 50 instead of 10 of [23] so that a clearer image of the iSHM class maps and the following iSHM footprints can be examined. The respective isodynamic capacity chart of Fig. 1(a) is plotted in Fig. 1(b) without considering the borderlines between OV LV BPL topology classes.

With reference to Figs. 1(a) and 1(b), several interesting remarks can be pointed out concerning the location of the modified OV LV BPL topologies and the inner class area capacity distribution of the iSHM class maps, namely:

- By comparing Fig. 1(b) with Fig. 3(a) of [20], it is evident that the iSHM footprint of OV LV BPL topologies with one branch is clearly constrained in the rural class area while the majority of the cases examined are approximately located at the same isodynamic capacity curve with small right or left deviations. Here it should be reminded that the OV LV BPL topologies with longer branches tend to present increased values of $\hat{a}_{\mathrm{MLE}}^{\mathrm{Weibull}}$ and $\hat{\beta}_{\mathrm{MLE}}^{\mathrm{Weibull}}$ and thus are located to the upper right part of the isodynamic capacity curve while a small right shift from the isodynamic capacity curve is expected since the OV LV BPL topologies with longer branches are characterized by relatively lower capacities in comparison with the real indicative OV LV BPL rural case. The opposite situation holds for the OV LV BPL topologies with one short branch. In the case of OV LV BPL topologies with two branches, similar observations can be made but for the suburban class area and for its isodynamic capacity curves.

- As the effect of branch line faults is examined and the potential of the branch line fault detection is discussed, the iSHM footprint of OV LV BPL topologies with one branch line fault is not strictly located at adjacent isodynamic capacity curves as shown by comparing Fig. 1(b) with Fig. 5 of [20]. In fact, the identification of the OV LV BPL topologies with branch line faults of short length becomes easier since their corresponding iSHM footprint violates the class area borders in accordance with [20]. The violation of the class area borders is anyway an easy task for the management information system of the smart grid to detect. 


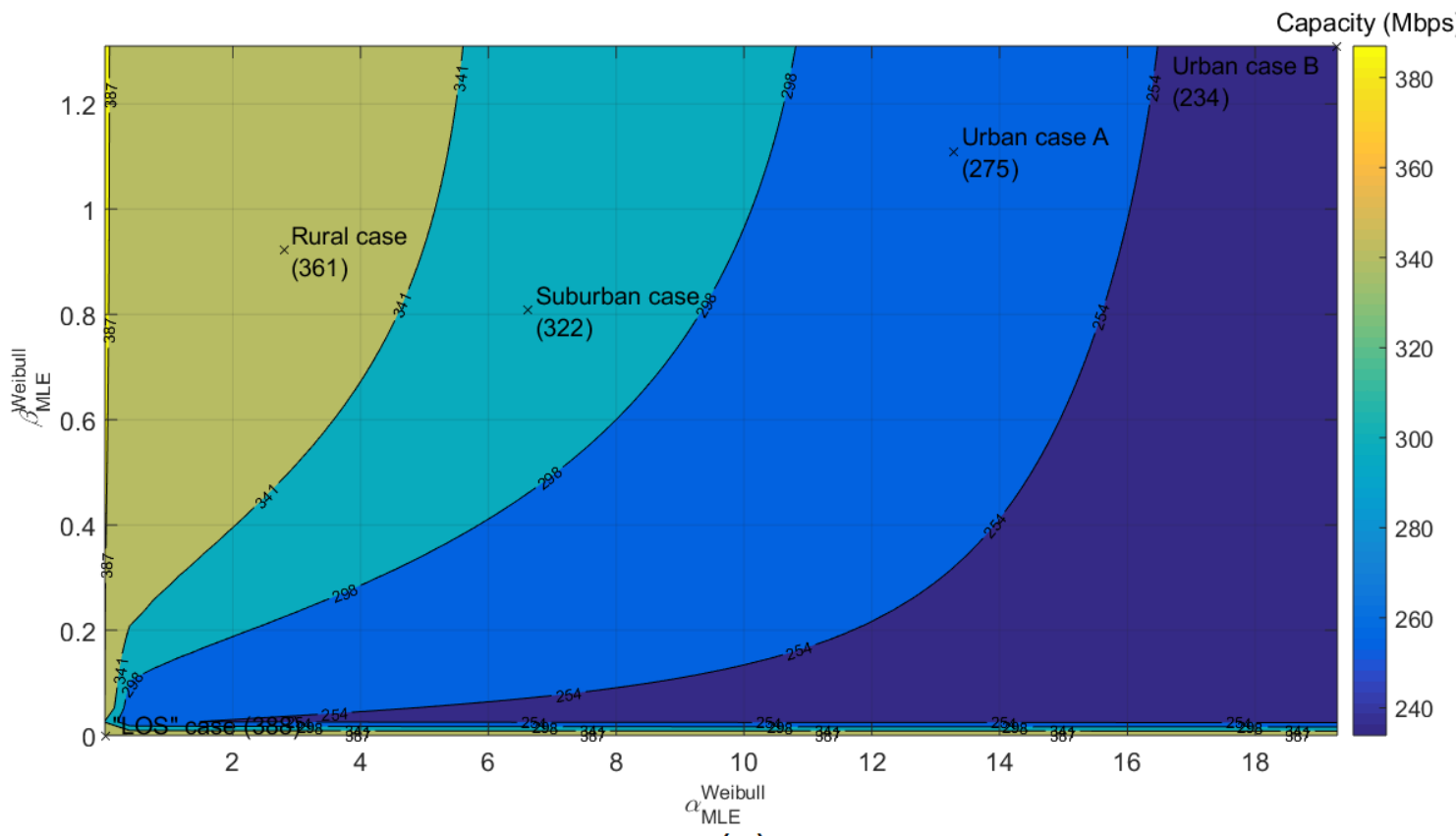

(a)

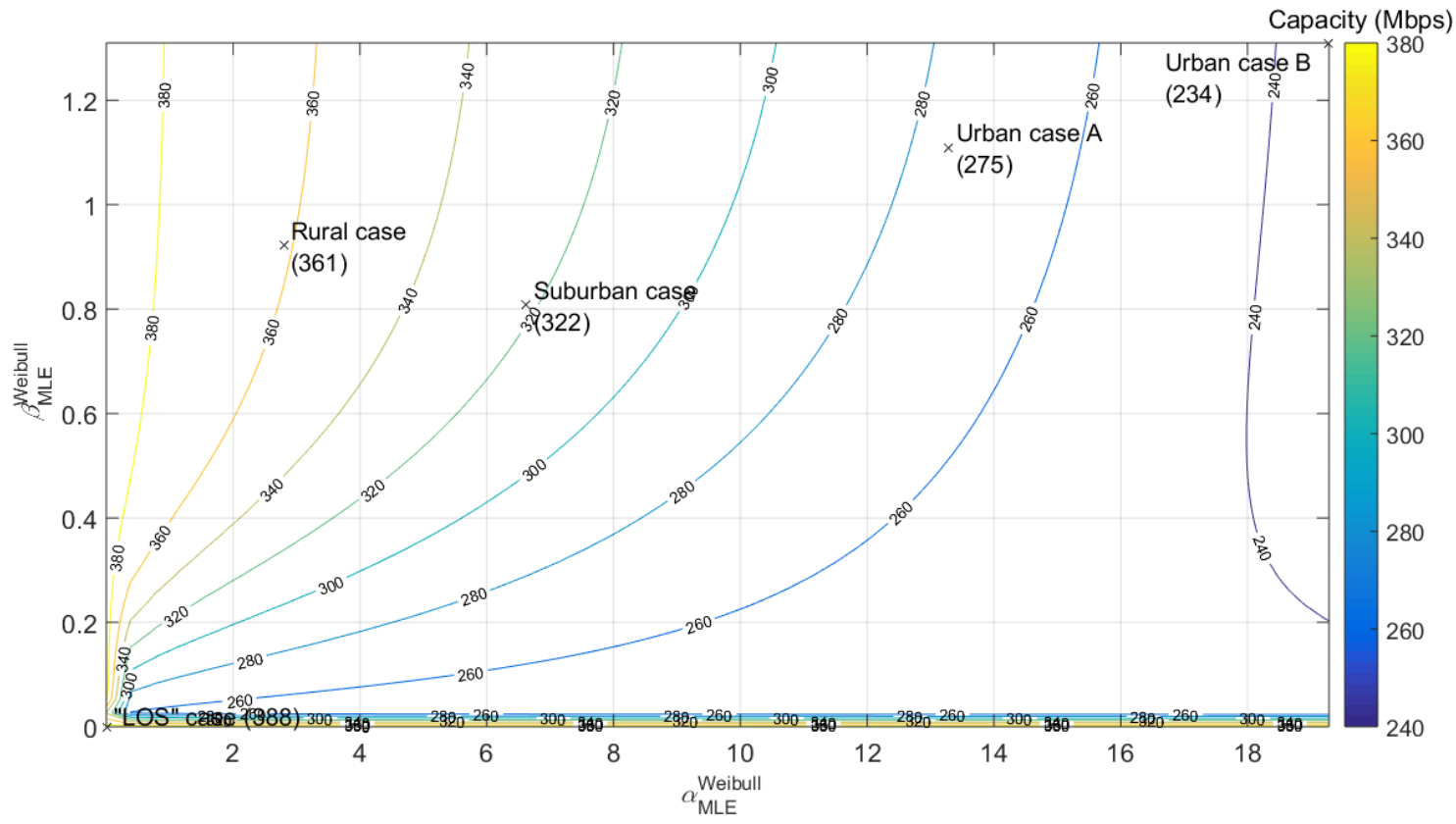

(b)

Fig. 1. (a) iSHM class map of OV LV BPL topologies when $3-30 \mathrm{MHz}$ frequency band, $0.1 \mathrm{MHz}$ frequency subchannel spacing, $\mathrm{WtG}^{1}$ coupling scheme and FCC Part 15 are assumed [20]. (b) Isodynamic capacity chart of Fig.1(a) with isodynamic capacity curves.

The same result is reached by comparing the location of white spots of the iSHM footprint of OV LV BPL topologies with one branch line fault with the isodynamic capacity curves.

- As the effect of single hooks for energy theft is examined and their detection potential is studied, the iSHM footprint of OV LV BPL topologies with one hook 
for energy theft is significantly right shifted from the real indicative OV LV BPL topology of reference. Since the vast majority of the white spots of the iSHM footprint of OV LV BPL topologies with one hook for energy theft is dislocated and approximately located on an isodynamic capacity curve far right, this implies that the detection of energy thefts is far easier by the management information system than the detection of branch line faults.

With reference to Fig. 1(b), it is clearly shown that the inner class area capacity distribution of the iSHM class maps remains zonal and solid without islands of capacity changes. The violation of isodynamic capacity curves helps towards the identification of a critical event during the operation of the power grid while the Weibull CASD MLE parameter pair can help towards the exact location of the critical event when the nature of the critical situation is identified by the management information system given the low intensity of the measurement differences.

But the aforementioned identification and classification of the critical situation presumes that the capacity of the modified OV LV BPL topology that suffers from the critical event is equal to the capacity of the applied isodynamic capacity curve which is plotted on the basis of the average capacities of the OV LV BPL topology subclass of Weibull CASD iSHM class map. For that reason, the capacity of the modified OV LV BPL topologies is compared with the average capacity of the OV LV BPL topology subclass of Weibull CASD iSHM class maps whose CASD MLE parameter pair is the same with the one of the examined modified OV LV BPL topology.

Note that the presentation of the topological characteristics of OV LV BPL topologies of the following Sections in this paper is made on the basis of the scheme of the typical OV LV BPL topology that is illustrated in Fig. 1(b) of [23]. Also, the topological characteristics of the five indicative OV LV BPL topologies, which act as the representative topologies of the respective classes and their main subclasses, are reported in Table 1 of [23] and on that basis the presentation of the topological characteristics of the arbitrary OV LV BPL topologies of the following Sections is given in the following Tables.

\section{2 iSHM Footprints and Capacity Differences of the Real OV LV BPL Topologies for the Default Operation Settings}

In Sec.2.4 of [20], the footprint of the real OV LV BPL topologies has been illustrated on the iSHM class maps where real OV LV BPL topologies have been retrieved by the Topology Identification Methodology (TIM) database of [25]. As the applied TIM BPL topology database specifications are concerned, they have been reported in [25] for the database preparation. For the further analysis of this Section, 12 arbitrary real OV LV BPL topologies of one branch and 16 arbitrary real OV LV BPL topologies of two branches, which are reported in Table 1 and 2, respectively, are assumed.

As the iSHM footprint parameters of the arbitrary real OV LV BPL topologies with one branch are concerned, Weibull CASD MLE parameter pairs (say, $\hat{a}_{\mathrm{MLE}}^{\text {Weibull }}$ and $\hat{\beta}_{\mathrm{MLE}}^{\text {Weibull }}$ ) of each of the 12 arbitrary real OV LV BPL topologies of one branch are reported in Table 1 as well as the capacity of the examined OV LV BPL topology. Also, the average capacity of the OV LV BPL topology subclass of Weibull CASD iSHM class map for given arbitrary real OV LV BPL topology of one branch is 
Table 1

Real OV LV BPL Topologies of one Branch, Weibull CASD MLEs and Capacities

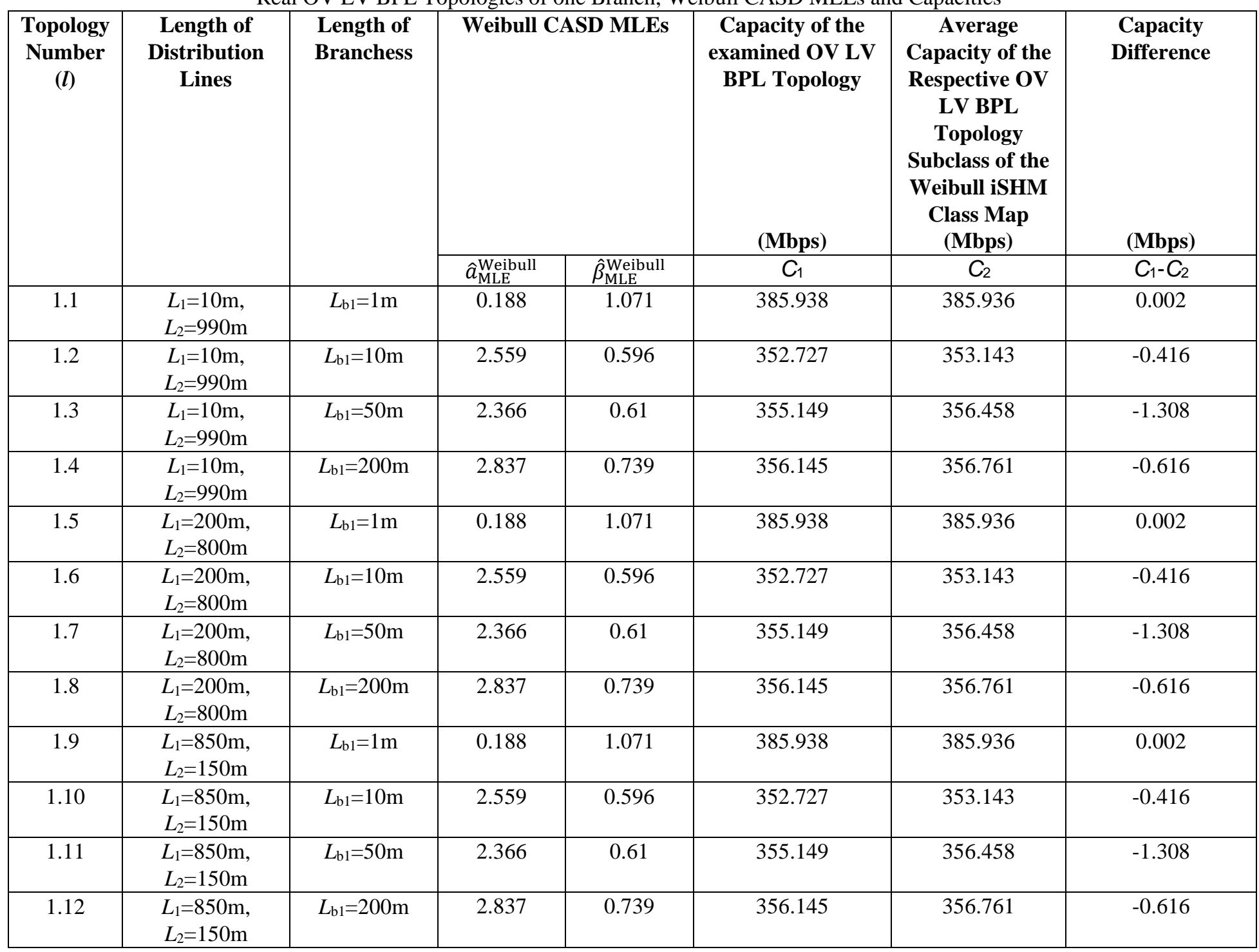


Table 2

Real OV LV BPL Topologies of two Branches, Weibull CASD MLEs and Capacities

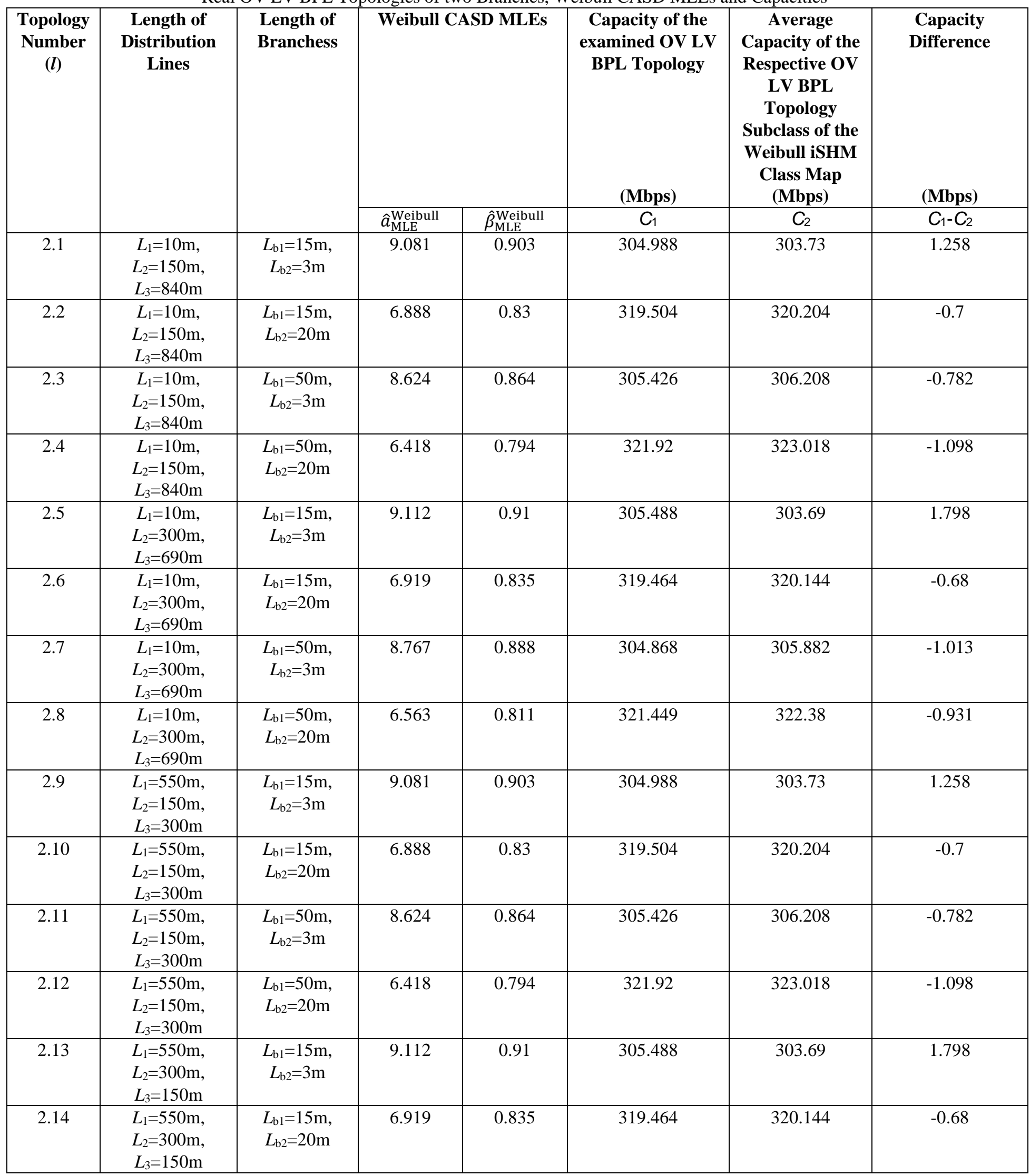




\begin{tabular}{|c|c|c|c|c|c|c|c|}
\hline 2.15 & $\begin{array}{l}L_{1}=550 \mathrm{~m}, \\
L_{2}=300 \mathrm{~m}, \\
\end{array}$ & $\begin{array}{c}L_{\mathrm{b} 1}=50 \mathrm{~m}, \\
L_{\mathrm{b} 2}=3 \mathrm{~m}\end{array}$ & 8.767 & 0.888 & 304.868 & 305.882 & -1.013 \\
\hline 2.16 & $L_{1}=550 \mathrm{~m}$, & $L_{\mathrm{b} 1}=50 \mathrm{~m}$, & 6.563 & 0.811 & 321.449 & 322.38 & -0.931 \\
& $L_{2}=300 \mathrm{~m}$, \\
& $L_{3}=150 \mathrm{~m}$ & $L_{\mathrm{b} 2}=20 \mathrm{~m}$ & & & & & \\
\hline
\end{tabular}

demonstrated as well as the capacity difference between the examined OV LV BPL topology and the average capacity of the OV LV BPL topology subclass of Weibull CASD iSHM class map of the same Weibull CASD MLE parameter pair with the one of the examined OV LV BPL topology. In Table 2, the same analytics with Table 1 are shown but for the case of the 16 arbitrary real OV LV BPL topologies of two branches.

By analyzing Tables 1 and 2, several important observations can be made concerning the iSHM footprints of the real OV LV BPL topologies and the inner class area capacity distribution of the iSHM class maps, namely:

- With reference to Weibull CASD MLEs, all the arbitrary OV LV BPL topologies of Table 1 and 2 are located at the rural and suburban class areas, respectively. This quantitative observation agrees with the graphical findings of iSHM footprints highlighted at Figs. 3(a) and 3(b) of [20].

- In Table 1, all the capacities of the arbitrary OV LV BPL topologies of one branch remain comparable except for Topologies 1.1, 1.5 and 1.9, which are characterized by the short branches of $1 \mathrm{~m}$. The almost equal capacities of the aforementioned arbitrary OV LV BPL topologies imply that their iSHM footprint lies near the same isodynamic capacity curve of 355Mbps. Same capacity results occur in Table 2 where the arbitrary OV LV BPL topologies of two branches are grouped to three isodynamic capacity curves.

- In accordance with Table 2 of [20], Weibull CASD succeeds in satisfying the strict capacity performance criteria of absolute threshold of percentage change and average absolute percentage change for the five indicative OV LV BPL topologies. In Table 1, it is shown that the capacity of the examined arbitrary OV LV BPL topology with one branch is approximately the same with the average capacity of the respective OV LV BPL topology subclass of the Weibull iSHM class map of Fig. 1(a) (say, capacity differences that range from $-1.308 \mathrm{Mbps}$ to $0.002 \mathrm{Mbps}$ ) that entails that Weibull CASD can accurately approximate the capacity of each OV LV BPL topology of one branch given the Weibull CASD MLEs. Same results can be observed in the case of OV LV BPL topologies with two branches where the capacity differences range from $-1.098 \mathrm{Mbps}$ to $1.798 \mathrm{Mbps}$. Therefore, Weibull CASD can produce successful capacity estimations for any OV LV BPL topology that lies at the rural and suburban class area given the Weibull CASD MLEs.

It is evident from the analysis of this Section that a direct correspondence can be assumed between Weibull CASD MLEs of an examined OV LV BPL topology, its position on the class map, its position on the isodynamic capacity curves and its capacity. Combined with the zonal capacity distribution behavior of class maps, it is shown that OV LV BPL topologies with one, two and three and above branches are expected to be located at rural, suburban and urban class areas, respectively. 
3.3 iSHM Footprints and Capacity Differences of the Real OV LV BPL Topologies with One Branch Line Fault for the Default Operation Settings

In Sec.2.5 of [20], the footprint of the real OV LV BPL topologies with one branch line fault has been illustrated on the iSHM class maps where the real indicative OV LV BPL urban case A has acted as the reference topology. Actually, Fault and Instability Identification Methodology (FIIM) database of [25] has been exploited so that all the possible real OV LV BPL topologies with one branch line fault can be retrieved by the real indicative OV LV BPL urban case A. For the further analysis of this Section, 6 arbitrary real OV LV BPL topologies of one branch line fault, which are based on the topological characteristics of the real indicative OV LV BPL urban case A, are reported in Table 3. Note that two different branch line fault lengths are assumed per each of the three branch lines of the real indicative urban case A.

As the iSHM footprint parameters of the arbitrary real OV LV BPL topologies with one branch line fault are concerned, Weibull CASD MLE parameter pair of each of the 6 arbitrary real OV LV BPL topologies of one branch line fault is reported in Table 3 as well as the capacity of the examined OV LV BPL topology. Similarly to Tables 1 and 2 , the average capacity of the OV LV BPL topology subclass of Weibull CASD iSHM class map for given arbitrary real OV LV BPL topology of one branch line fault and the capacity difference between the examined OV LV BPL topology and the average capacity of the OV LV BPL topology subclass of Weibull CASD iSHM class map of the same Weibull CASD MLE parameter pair with the one of the examined OV LV BPL topology are also given.

With respect to Figs. 1(a), 1(b) and Fig. 5 of [20], Table 3 may offer valuable information towards the detection of the critical event of branch line faults during the operation of the power grid by exploiting the iSHM footprints of the real OV LV BPL topologies and the inner class area capacity distribution of the iSHM class maps. More specifically:

- Similarly to Tables 1 and 2, the capacities of the examined arbitrary OV LV BPL topologies with one branch line fault remain approximately the same with the average capacities of the respective OV LV BPL topology subclasses of the Weibull iSHM class map of Fig. 1(a) (say, capacity differences that range from $3.604 \mathrm{Mbps}$ to $1.959 \mathrm{Mbps})$. The capacity differences are small enough in comparison with the achieved capacities of the examined OV LV BPL topologies that again entails that Weibull CASD can accurately approximate the capacity of each OV LV BPL topology of one branch line fault given its Weibull CASD MLEs.

- Since a direct correspondence between the Weibull CASD MLE parameter pair and the capacity of an examined OV LV BPL topology with one branch line fault is verified, there is no need for depicting the Weibull CASD MLE parameter pair of the examined OV LV BPL topology on the class map since a simple check of the achieved capacity of the examined OV LV BPL topology by the management information system with the capacity borderlines of a class area can assure if its location lies inside the class area or not. 
Table 3

OV LV BPL Urban Case A with one Branch Line Fault, Weibull CASD MLEs and Capacities

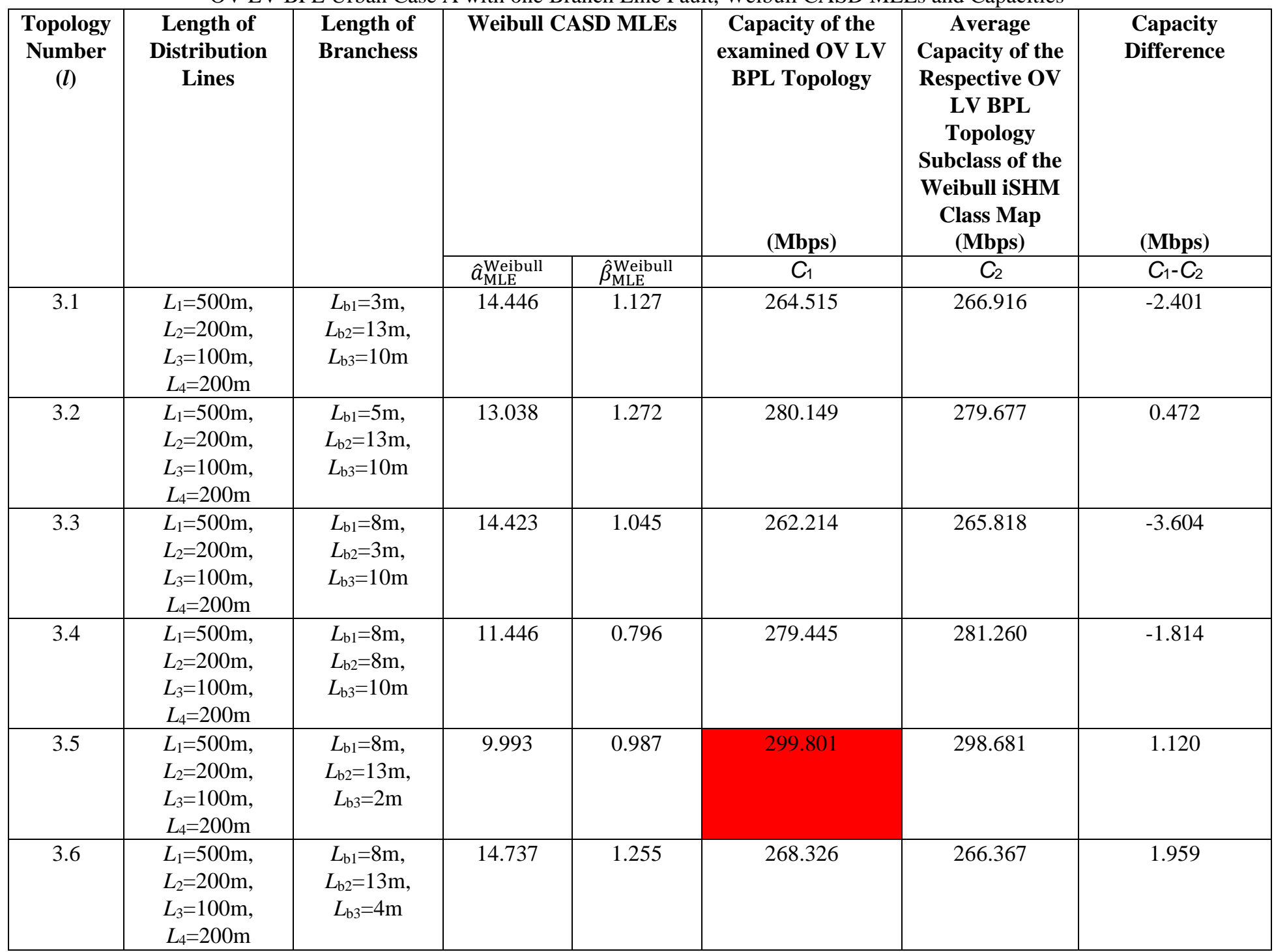

- Since the real indicative OV LV BPL urban case A acts as the basis for the study of single branch line faults of this Section, the capacities of the class area capacity borderlines are equal to $254 \mathrm{Mbps}$ and $298 \mathrm{Mbps}$. By examining the Table 3 , it is evident that Topology 3.5 presents capacity that is equal to $299.801 \mathrm{Mbps}$ and exceeds the upper capacity borderline of the urban case A class area. If a branch line fault is suspected during the operation of the power grid, this exceedance may be an alert for the management information system. Indeed, the capacity borderline violation remains the safest way to detect a potential branch line faults as also explained and depicted in Fig. 5 of [20].

- In accordance with Table 1 of [20] and Fig. 1(a), the real indicative OV LV BPL urban case $\mathrm{A}$ is characterized by $\hat{a}_{\mathrm{MLE}}^{\mathrm{Weibull}}, \hat{\beta}_{\mathrm{MLE}}^{\mathrm{Weibull}}$ and capacity that are equal to 13.29, 1.11 and 275Mbps, respectively. As the OV LV BPL topologies of Table 3 are regarded, their $\hat{a}_{\mathrm{MLE}}^{\mathrm{Weibull}}$ and $\hat{\beta}_{\mathrm{MLE}}^{\mathrm{Weibull}}$ range from 9.993 to 14.737 and from 
0.796 to 1.272 , respectively, while their capacities range from $266.367 \mathrm{Mbps}$ to 298.681Mbps. Since branch line fault reforms the existing affected branch line of the OV LV BPL topology to a shorter one, capacity fluctuations can be observed while a movement across the isodynamic capacity curve is expected due to the fluctuations of $\hat{a}_{\mathrm{MLE}}^{\mathrm{Weibull}}$ and $\hat{\beta}_{\mathrm{MLE}}^{\mathrm{Weibull}}$.

As the violation of class area capacity borderlines and of the isodynamic capacity curves is an evidence for the existence of a branch line fault when no measurement differences are assumed, FIIM, which is analyzed in [25], can be then activated so that the branch line fault can securely identified through its supported repertory of faults and instabilities. Anyway, FIIM is a part of the management information system of the smart grid and here its performance is enhanced. As already been identified, the detection of branch line fault of short length becomes an easier task since violation of the capacity borderlines of the corresponding class area is more expected.

\section{4 iSHM Footprints and Capacity Differences of the Real OV LV BPL Topologies with One Hook for Energy Theft for the Default Operation Settings}

In Sec. 2.6 of [20], the footprint of the real OV LV BPL topologies with a single hook for energy theft has been shown on the iSHM class maps where the real indicative OV LV BPL suburban case has acted as the basis topology. In accordance to [26], Hook Style energy theft DETection (HS-DET) method may generate all the real OV LV BPL topologies with a single hook for energy theft that are based on the OV LV BPL suburban case but for the sake of the analysis of this Section, 9 arbitrary real OV LV BPL topologies of one hook for energy theft, which are based on the topological characteristics of the real indicative OV LV BPL suburban case, are reported in Table 4.

As the iSHM footprint parameters of the arbitrary real OV LV BPL topologies with one hook for energy theft are concerned, Weibull CASD MLE parameter pair of each of the 9 arbitrary real OV LV BPL topologies of one hook for energy theft is reported in Table 4 as well as the capacity of the examined OV LV BPL topology. Similarly to Tables 1-3, the average capacity of the OV LV BPL topology subclass of Weibull CASD iSHM class map for given arbitrary real OV LV BPL topology of one hook for energy theft and the capacity difference between the examined OV LV BPL topology and the average capacity of the OV LV BPL topology subclass of Weibull CASD iSHM class map of the same Weibull CASD MLE parameter pair with the one of the examined OV LV BPL topology are also given. 
Table 4

OV LV BPL Suburban Case with one Single Hook for Energy Theft, Weibull CASD MLEs and Capacities

\begin{tabular}{|c|c|c|c|c|c|c|c|}
\hline \multirow[t]{2}{*}{$\begin{array}{l}\text { Topology } \\
\text { Number } \\
(l)\end{array}$} & \multirow[t]{2}{*}{$\begin{array}{c}\text { Length of } \\
\text { Distribution } \\
\text { Lines }\end{array}$} & \multirow[t]{2}{*}{$\begin{array}{l}\text { Length of } \\
\text { Branchess }\end{array}$} & Weibull & D MLEs & \multirow[t]{2}{*}{$\begin{array}{c}\text { Capacity of the } \\
\text { examined OV LV } \\
\text { BPL Topology } \\
\\
(\mathrm{Mbps}) \\
C_{1}\end{array}$} & \multirow[t]{2}{*}{$\begin{array}{c}\text { Average } \\
\text { Capacity of the } \\
\text { Respective OV } \\
\text { LV BPL } \\
\text { Topology } \\
\text { Subclass of the } \\
\text { Weibull iSHM } \\
\text { Class Map } \\
\text { (Mbps) } \\
C_{2}\end{array}$} & \multirow[t]{2}{*}{$\begin{array}{c}\text { Capacity } \\
\text { Difference }\end{array}$} \\
\hline & & & $\hat{a}_{\mathrm{MLE}}^{\text {Weibull }}$ & $\hat{\beta}_{\mathrm{MLE}}^{\text {Weibull }}$ & & & \\
\hline 4.1 & $\begin{array}{c}L_{1}=1 \mathrm{~m}, \\
L_{2}=499 \mathrm{~m}, \\
L_{3}=400 \mathrm{~m}, \\
L_{4}=100 \mathrm{~m}\end{array}$ & $\begin{array}{c}L_{\mathrm{b} 1}=3 \mathrm{~m}, \\
L_{\mathrm{b} 2}=50 \mathrm{~m}, \\
L_{\mathrm{b} 3}=10 \mathrm{~m}\end{array}$ & 13.564 & 1.057 & 272.983 & 272.305 & 0.678 \\
\hline 4.2 & $\begin{array}{c}L_{1}=1 \mathrm{~m}, \\
L_{2}=499 \mathrm{~m}, \\
L_{3}=400 \mathrm{~m}, \\
L_{4}=100 \mathrm{~m}\end{array}$ & $\begin{array}{l}L_{\mathrm{b} 1}=15 \mathrm{~m}, \\
L_{\mathrm{b} 2}=50 \mathrm{~m}, \\
L_{\mathrm{b} 3}=10 \mathrm{~m}\end{array}$ & 11.463 & 1.006 & 287.521 & 287.316 & 0.205 \\
\hline 4.3 & $\begin{array}{c}L_{1}=1 \mathrm{~m}, \\
L_{2}=499 \mathrm{~m}, \\
L_{3}=400 \mathrm{~m}, \\
L_{4}=100 \mathrm{~m}\end{array}$ & $\begin{array}{c}L_{\mathrm{b} 1}=100 \mathrm{~m}, \\
L_{\mathrm{b} 2}=50 \mathrm{~m}, \\
L_{\mathrm{b} 3}=10 \mathrm{~m}\end{array}$ & 11.196 & 1.078 & 291.723 & 291.085 & 0.638 \\
\hline 4.4 & $\begin{array}{l}L_{1}=200 \mathrm{~m}, \\
L_{2}=300 \mathrm{~m}, \\
L_{3}=400 \mathrm{~m}, \\
L_{4}=100 \mathrm{~m}\end{array}$ & $\begin{array}{c}L_{\mathrm{b} 1}=3 \mathrm{~m}, \\
L_{\mathrm{b} 2}=50 \mathrm{~m}, \\
L_{\mathrm{b} 3}=10 \mathrm{~m}\end{array}$ & 13.501 & 1.069 & 273.302 & 272.997 & 0.305 \\
\hline 4.5 & $\begin{aligned} L_{1} & =200 \mathrm{~m}, \\
L_{2} & =300 \mathrm{~m}, \\
L_{3} & =400 \mathrm{~m}, \\
L_{4} & =100 \mathrm{~m}\end{aligned}$ & $\begin{array}{l}L_{\mathrm{b} 1}=15 \mathrm{~m}, \\
L_{\mathrm{b} 2}=50 \mathrm{~m}, \\
L_{\mathrm{b} 3}=10 \mathrm{~m}\end{array}$ & 11.289 & 0.998 & 288.358 & 288.498 & -0.140 \\
\hline 4.6 & $\begin{array}{l}L_{1}=200 \mathrm{~m}, \\
L_{2}=300 \mathrm{~m}, \\
L_{3}=400 \mathrm{~m}, \\
L_{4}=100 \mathrm{~m}\end{array}$ & $\begin{array}{c}L_{\mathrm{b} 1}=100 \mathrm{~m}, \\
L_{\mathrm{b} 2}=50 \mathrm{~m}, \\
L_{\mathrm{b} 3}=10 \mathrm{~m}\end{array}$ & 11.053 & 1.057 & 292.244 & 291.770 & 0.474 \\
\hline 4.7 & $\begin{array}{l}L_{1}=500 \mathrm{~m}, \\
L_{2}=200 \mathrm{~m}, \\
L_{3}=200 \mathrm{~m}, \\
L_{4}=100 \mathrm{~m}\end{array}$ & $\begin{aligned} L_{\mathrm{b} 1} & =50 \mathrm{~m}, \\
L_{\mathrm{b} 2} & =3 \mathrm{~m}, \\
L_{\mathrm{b} 3} & =10 \mathrm{~m}\end{aligned}$ & 13.438 & 1.044 & 272.895 & 272.996 & -0.102 \\
\hline 4.8 & $\begin{array}{l}L_{1}=500 \mathrm{~m}, \\
L_{2}=200 \mathrm{~m}, \\
L_{3}=200 \mathrm{~m}, \\
L_{4}=100 \mathrm{~m}\end{array}$ & $\begin{array}{l}L_{\mathrm{b} 1}=50 \mathrm{~m}, \\
L_{\mathrm{b} 2}=15 \mathrm{~m}, \\
L_{\mathrm{b} 3}=10 \mathrm{~m}\end{array}$ & 11.271 & 0.977 & 287.904 & 288.103 & -0.199 \\
\hline 4.9 & $\begin{array}{l}L_{1}=500 \mathrm{~m}, \\
L_{2}=200 \mathrm{~m}, \\
L_{3}=200 \mathrm{~m}, \\
L_{4}=100 \mathrm{~m}\end{array}$ & $\begin{array}{c}L_{\mathrm{b} 1}=50 \mathrm{~m}, \\
L_{\mathrm{b} 2}=100 \mathrm{~m}, \\
L_{\mathrm{b} 3}=10 \mathrm{~m}\end{array}$ & 11.170 & 1.092 & 292.164 & 291.580 & 0.585 \\
\hline
\end{tabular}


With respect to Figs. 1(a), 1(b) and Fig. 7 of [20], Table 4 verifies the convenience to detect the energy theft by the management information system of the smart grid. Since the hook of energy theft may be treated as an additional branch line to the existing ones of the examined OV LV BPL topology, this implies the transition of the examined OV LV BPL topology from the existing topology class to the one more aggravated; say, in the case of this Section, the original OV LV BPL topology is located at the suburban class area while the modified ones after the insertion of hook (i.e., Topologies 4.1-4.9) are located at the class area of the urban case A. By taking into account the capacity of the modified OV LV BPL topologies, the low capacity differences and the capacity borderlines of the urban case A class area, it is evident that a steep decrease of the capacity that occurs due to the insertion of the hook means a possible energy theft if all other critical events are eliminated. Another iSHM footprint characteristic that has been highlighted in Table 4 is the steep and steady increase of $\hat{a}_{\mathrm{MLE}}^{\mathrm{Weibull}}$ with respect to $\hat{a}_{\mathrm{MLE}}^{\mathrm{Weibull}}$ of OV LV BPL suburban case, which is equal to 6.62 as reported in Table 1 of [20]. Also an increase of $\hat{\beta}_{\mathrm{MLE}}^{\mathrm{Weibull}}$ can also been observed in modified OV LV BPL topologies but this occurs in the majority of the cases as presented in Fig.7 of [20]. Due to the generalized differentiation of $\hat{a}_{\mathrm{MLE}}^{\mathrm{Weibull}}, \hat{\beta}_{\mathrm{MLE}}^{\mathrm{Weibull}}$ and capacity, the detection of the hook style energy theft remains easier than the one of branch line fault while the application of HS-DET method as analyzed in [26] may allow the location determination of any hook style energy theft in OV LV BPL networks.

\subsection{Weibull iSHM Class Maps and Inner Class Area Capacity Distribution Rules of Thumb}

As already been mentioned for Figs. 1(a) and 1(b), first, either Weibull iSHM topology class borderlines or isodynamic capacity curves create solid capacity zones without capacity islands on them. Second, it has been proven in Tables 1-4 that the capacity of the examined OV LV BPL topologies remains almost equal to the average capacity of the respective OV LV BPL topology subclasses of Weibull CASD iSHM class map for given Weibull CASD MLE parameter pair. Third, either Weibull iSHM topology class borderlines or isodynamic capacity curves can be satisfactorily approximated by equations that involve $\hat{a}_{\mathrm{MLE}}^{\mathrm{Weibull}}$ and $\hat{\beta}_{\mathrm{MLE}}^{\mathrm{Weibull}}$ thus clearly defining the limits so that (i) the examined OV LV BPL topology can be grouped among the available topology classes through its Weibull CASD MLE parameter pair; and (ii) the capacity of the examined OV LV BPL topology can be estimated through its Weibull CASD MLE parameter pair. For clarity issues, the following approximation analysis is focused: (i) between the $\hat{a}_{\mathrm{MLE}}^{\mathrm{Weibull}}$ and $\hat{\beta}_{\mathrm{MLE}}^{\mathrm{Weibull}}$ graphical limits of Figs. 1(a)-(b); and (ii) the between the $\hat{a}_{\mathrm{MLE}}^{\mathrm{Weibull}}$ and $\hat{\beta}_{\mathrm{MLE}}^{\mathrm{Weibull}}$ limits where topology class borderlines and isodynamic capacity curves remain smooth without breaks; say, $\hat{a}_{\mathrm{MLE}}^{\mathrm{Weibull}}$ ranges from 2 to 20 and $\hat{\beta}_{\mathrm{MLE}}^{\text {Weibull }}$ ranges from 0.2 to 1.2 .

More specifically, let assume that Weibull iSHM topology class borderlines of Fig. 1(a) and Weibull iSHM isodynamic capacity curves of Fig. 1(b) may be approximated through quadratic regression equations that are detailed in Tables 5 and 6, respectively. Now, let assume arbitrary OV LV BPL topologies, say, Topologies 1.1, 2.1, 3.1 and 4.1, whose $\hat{a}_{\mathrm{MLE}}^{\mathrm{Weibull}}$ and $\hat{\beta}_{\mathrm{MLE}}^{\mathrm{Weibull}}$ are alredy reported in Tables $1-4$, respectively. 
Table 5

Quadratic Regression Equations of iSHM Topology Class Borderlines of Fig. 1(a)

\begin{tabular}{|c|c|}
\hline $\begin{array}{c}\text { OV LV BPL } \\
\text { Topology Class } \\
\text { Capacity of the } \\
\text { Right Borderline } \\
\text { in Mbps) }\end{array}$ & Right Borderline Equation \\
\hline $\begin{array}{c}\text { Rural } \\
(341)\end{array}$ & $R_{5.1}=\hat{\beta}_{\mathrm{MLE}}^{\mathrm{Weibull}}-\left\{\left[0.0236 \cdot\left(\hat{a}_{\mathrm{MLE}}^{\mathrm{Weibull}}\right)^{2}\right]+\left[0.0304 \cdot \hat{a}_{\mathrm{MLE}}^{\mathrm{Weibull}}\right]+0.2174\right\}$ \\
\hline $\begin{array}{c}\text { Suburban } \\
(298)\end{array}$ & $R_{5.2}=\hat{\beta}_{\mathrm{MLE}}^{\mathrm{Weibull}}-\left\{\left[0.0133 \cdot\left(\hat{a}_{\mathrm{MLE}}^{\mathrm{Weibull}}\right)^{2}\right]+\left[-0.05 \cdot \hat{a}_{\mathrm{MLE}}^{\mathrm{Weibull}}\right]+0,1943\right\}$ \\
\hline $\begin{array}{c}\text { Urban Case A } \\
(254)\end{array}$ & $R_{5.3}=\hat{\beta}_{\mathrm{MLE}}^{\mathrm{Weibull}}-\left\{\left[0.0089 \cdot\left(\hat{a}_{\mathrm{MLE}}^{\mathrm{Weibull}}\right)^{2}\right]+\left[-0.1116 \cdot \hat{a}_{\mathrm{MLE}}^{\mathrm{Weibull}}\right]+0.3324\right\}$ \\
\hline
\end{tabular}

Table 6

Quadratic Regression Equations of iSHM isodynamic capacity curves of Fig. 1(b)

\begin{tabular}{|c|c|}
\hline $\begin{array}{c}\text { Capacity in } \\
\text { Mbps of the } \\
\text { Isodynamic } \\
\text { Curve }\end{array}$ & Isodynamic Capacity Curve Equation \\
\hline 360 & $R_{6.1}=\hat{\beta}_{\mathrm{MLE}}^{\mathrm{Weibull}}-\left\{\left[0.1228 \cdot\left(\hat{a}_{\mathrm{MLE}}^{\mathrm{Weibull}}\right)^{2}\right]+\left[-0.1581 \cdot \hat{a}_{\mathrm{MLE}}^{\mathrm{Weibull}}\right]+0.3878\right\}$ \\
\hline 340 & $R_{6.2}=\hat{\beta}_{\mathrm{MLE}}^{\mathrm{Weibull}}-\left\{\left[0.0391 \cdot\left(\hat{a}_{\mathrm{MLE}}^{\mathrm{Weibull}}\right)^{2}\right]+\left[-0.0703 \cdot \hat{a}_{\mathrm{MLE}}^{\mathrm{Weibull}}\right]+0.3144\right\}$ \\
\hline 320 & $R_{6.3}=\hat{\beta}_{\mathrm{MLE}}^{\mathrm{Weibull}}-\left\{\left[0.0198 \cdot\left(\hat{a}_{\mathrm{MLE}}^{\mathrm{Weibull}}\right)^{2}\right]+\left[-0.0387 \cdot \hat{a}_{\mathrm{MLE}}^{\mathrm{Weibull}}\right]+0.2319\right\}$ \\
\hline 300 & $R_{6.4}=\hat{\beta}_{\mathrm{MLE}}^{\mathrm{Weibull}}-\left\{\left[0.0141 \cdot\left(\hat{a}_{\mathrm{MLE}}^{\mathrm{Weibull}}\right)^{2}\right]+\left[-0.0608 \cdot \hat{a}_{\mathrm{MLE}}^{\mathrm{Weibull}}\right]+0.2419\right\}$ \\
\hline 280 & $R_{6.5}=\hat{\beta}_{\mathrm{MLE}}^{\mathrm{Weibull}}-\left\{\left[0.0112 \cdot\left(\hat{a}_{\mathrm{MLE}}^{\mathrm{Weibull}}\right)^{2}\right]+\left[-0.0696 \cdot \hat{a}_{\mathrm{MLE}}^{\mathrm{Weibull}}\right]+0.1897\right\}$ \\
\hline 260 & $R_{6.6}=\hat{\beta}_{\mathrm{MLE}}^{\mathrm{Weibull}}-\left\{\left[0.0081 \cdot\left(\hat{a}_{\mathrm{MLE}}^{\mathrm{Weibull}}\right)^{2}\right]+\left[-0.0715 \cdot \hat{a}_{\mathrm{MLE}}^{\mathrm{Weibull}}\right]+0.1553\right\}$ \\
\hline
\end{tabular}

Table 7

OV LV BPL Topologies and Quadratic Regression Equations of iSHM Topology Class Borderlines of

Table 5

\begin{tabular}{|c|c|c|c|c|c|c|}
\hline \multirow{2}{*}{$\begin{array}{l}\text { Topology } \\
\text { Number } \\
(l)\end{array}$} & \multicolumn{2}{|c|}{$\begin{array}{l}\text { Weibull CASD } \\
\text { MLEs }\end{array}$} & \multirow{2}{*}{$\begin{array}{c}\text { Capacity of } \\
\text { the examined } \\
\text { OV LV BPL } \\
\text { Topology } \\
\text { (Mbps) } \\
C_{1}\end{array}$} & \multicolumn{3}{|c|}{$\begin{array}{c}\text { iSHM Topology Class Right Borderline } \\
\text { Equation }\end{array}$} \\
\hline & $\hat{a}_{\mathrm{MLE}}^{\text {Weibull }}$ & $\hat{\beta}_{\mathrm{MLE}}^{\text {Weibull }}$ & & $\begin{array}{c}R_{5.1} \\
\text { (Rural / 341) }\end{array}$ & $\begin{array}{c}R_{5.2} \\
\text { (Suburban / } \\
298)\end{array}$ & $\begin{array}{c}R_{5.3} \\
\text { (Urban Case A / } \\
254)\end{array}$ \\
\hline 1.1 & 0.188 & 1.071 & 385.938 & 0.847051 & 0.88563 & 0.759266 \\
\hline 2.1 & 9.081 & 0.903 & 304.988 & -1.53663 & 0.065971 & 0.850105 \\
\hline 3.1 & 14.446 & 1.127 & 264.515 & -4.45457 & -1.12054 & 0.54946 \\
\hline 4.1 & 13.564 & 1.057 & 272.983 & -3.91472 & -0.90606 & 0.600902 \\
\hline
\end{tabular}


Table 8

OV LV BPL Topologies and Quadratic Regression Equations of iSHM Isodynamic Capacity Curves of Table 6

\begin{tabular}{|c|c|c|c|c|c|c|c|c|c|}
\hline \multirow{2}{*}{$\begin{array}{c}\text { Topology } \\
\text { Number } \\
(l)\end{array}$} & \multicolumn{2}{|c|}{$\begin{array}{c}\text { Weibull CASD } \\
\text { MLES }\end{array}$} & $\begin{array}{c}\text { Capacity } \\
\text { of the }\end{array}$ & \multicolumn{6}{|c|}{ iSHM Isodynamic Capacity Curve Equation } \\
\hline & $\hat{a}_{\mathrm{MLE}}^{\text {Weibull }}$ & $\hat{\beta}_{\mathrm{MLE}}^{\text {Weibull }}$ & $C_{1}$ & $\begin{array}{c}R_{6.1} \\
(360)\end{array}$ & $\begin{array}{c}R_{6.2} \\
(340)\end{array}$ & $\begin{array}{c}R_{6.3} \\
(320)\end{array}$ & $\begin{array}{c}R_{6.4} \\
(300)\end{array}$ & $\begin{array}{c}R_{6.5} \\
(280)\end{array}$ & $\begin{array}{l}R_{6.6} \\
(260)\end{array}$ \\
\hline 1.1 & 0.188 & 1.071 & 385.938 & 0.649783 & 0.636234 & 0.630976 & 1.014192 & 0.637089 & 0.637556 \\
\hline 2.1 & 9.081 & 0.903 & 304.988 & -8.23454 & -2.12957 & -0.82496 & 0.109542 & 0.164835 & 0.437729 \\
\hline 3.1 & 14.446 & 1.127 & 264.515 & -22.6624 & -6.4637 & -2.89254 & -2.01022 & -0.65145 & 0.022925 \\
\hline 4.1 & 13.564 & 1.057 & 272.983 & -19.8381 & -5.62975 & -2.50752 & -1.49095 & -0.50615 & 0.089971 \\
\hline
\end{tabular}

On the basis of $\hat{a}_{\mathrm{MLE}}^{\mathrm{Weibull}}$ and $\hat{\beta}_{\mathrm{MLE}}^{\mathrm{Weibull}}$ of each of the aforementioned four topologies, the computation results of the application of iSHM topology class right borderline quadratic regression equations $R_{5.1}-R_{5.3}$ are demonstrated in Table 7 as well as the capacity of each OV LV BPL topology. Similar results with Table 7 are given in Table 8 but for the case of iSHM isodynamic capacity curve quadratic regression equations $R_{6.1}-R_{6.6}$.

By observing Tables 7 and 8, two rules of thumb that are based on the inner class area capacity distribution are first presented in the following analysis. In order to define these rules of thumb, an evidence that is very useful during the following analysis is the last positive value of quadratic regression equation results per each row (i.e., per each examined OV LV BPL topology) from right to left that is filled with green color in Tables 7 and 8. As for the two rules of thumb that are proposed, the first one has to do with the classification of OV LV BPL topologies while the second one has to with the approximate computation of the OV LV BPL topology capacity, namely:

- First rule of thumb concerning the OV LV BPL topology classification: As the inner class area capacity distribution suggests, the class areas are solid and are bounded by borderlines approximated by the equations of Table 5. In accordance with Table 7, the positive values for given OV LV BPL topology imply that the respective quadratic regression equations lie below the Weibull CASD MLEs of the examined OV LV BPL topology. The opposite fact holds when negative values occur. Since quadratic regression equations of Table 7 describe the right borderlines of the class areas and, hence, the respective lower capacity bounds of the class areas, the last positive value per row in Table 7 determines the OV LV BPL topology class of the examined topology. Therefore, Topology 1.1, 2.1, 3.1 and 4.1 are members of the rural, suburban, urban case A and urban case A OV LV BPL topology classes, respectively. The last remark is easily verified by comparing the capacity of the examined topology with the capacities of right borderlines of the OV LV BPL topology classes that are reported in Table 7; e.g., Topology 1.1 capacity is equal to $385.938 \mathrm{Mbps}$ that is greater than the lower 
capacity bound of rural class, which is equal to $341 \mathrm{Mbps}$, and lower than the "LOS" class capacity of $387 \mathrm{Mbps}$, which is presented in Fig. 1(a) and only consists of the "LOS" case. Same observations stand for the Topologies 2.1, 3.1 and 4.1 as well as for the vast majority of the OV LV BPL topologies described by Weibull CASD MLEs.

- Second rule of thumb concerning the capacity approximation of $O V L V B P L$ topology: Similarly to the first rule of thumb, isodynamic capacity curves bound compact capacity areas while the equations of the iSHM isodynamic capacity curves are given in Table 6 . In accordance with Table 8 , the positive values for given OV LV BPL topology imply that the respective quadratic regression equations lie below the Weibull CASD MLEs of the examined OV LV BPL topology while the negative values imply that the respective quadratic regression equations lie above. Similarly to the first rule of thumb, the last positive value per row in Table 8 may approximate the OV LV BPL topology capacity with lower and upper capacity bounds the capacity of the current isodynamic capacity curve of the last positive value and the capacity of the next isodynamic capacity curve, respectively. The last approximation is easily verified by comparing the capacity of the examined topology with the capacities of the isodynamic capacity curves that are reported in Table 8; e.g., Topology 2.1 capacity is equal to $304.988 \mathrm{Mbps}$ that is greater than the lower capacity bound defined by the isodynamic capacity curve $R_{6.4}$, which is equal to $300 \mathrm{Mbps}$, and lower than the upper capacity bound defined by the isodynamic capacity curve $R_{6.3}$, which is equal to $320 \mathrm{Mbps}$. Same observations stand for the Topologies 1.1, 3.1 and 4.1 as well as for the vast majority of the OV LV BPL topologies characterized by Weibull CASD MLEs.

\section{Conclusions}

In this paper, the study of the inner class area capacity distribution of Weibull iSHM class maps has allowed the better understanding of the relation between Weibull CASD MLEs and the capacity of the OV LV BPL topologies. Actually, regardless of the operation conditions of the OV LV power grid (either normal operation or faulty operation), Weibull CASD MLEs permit the capacity estimation of the examined OV LV BPL topologies. By exploiting the solid capacity areas of the Weibull iSHM class maps and iSHM footprints of the OV LV BPL topologies during their fault operation, critical events such as the branch line faults and the energy thefts can be easily detected through the careful consideration of iSHM footprints of the modified OV LV BPL topologies by the management information system of the OV LV BPL networks. Going one step further, two rules of thumb have been proposed that allow the classification and the capacity approximation of the OV LV BPL topologies by exploiting class area borderline and isodynamic capacity curve quadratic regression equations, respectively. 


\section{CONFLICTS OF INTEREST}

The author declares that there is no conflict of interests regarding the publication of this paper.

\section{References}

[1] Y. J. A. Zhang, H. P. Schwefel, H. Mohsenian-Rad, C. Wietfeld, C. Chen, and H. Gharavi, "Guest Editorial Special Issue on Communications and Data Analytics in Smart Grid," IEEE Journal on Selected Areas in Communications, vol. 38, no. 1, pp. 1-4, 2020.

[2] A. G. Lazaropoulos, "Wireless Sensor Network Design for Transmission Line Monitoring, Metering and Controlling Introducing Broadband over PowerLinesenhanced Network Model (BPLeNM)," ISRN Power Engineering, vol. 2014, Article ID 894628, 22 pages, 2014. doi:10.1155/2014/894628. [Online]. Available:

http://www.hindawi.com/journals/isrn.power.engineering/2014/894628/

[3] A. M. Tonello and T. Zheng, "Bottom-up transfer function generator for broadband PLC statistical channel modeling," In Proc. IEEE International Symposium on Power Line Communications and Its Applications 2009, ISPLC 2009, pp. 7-12, Mar. 2009.

[4] A. M. Tonello and F. Versolatto, "Bottom-up statistical PLC channel modelingPart I: Random topology model and efficient transfer function computation," IEEE Transactions on Power Delivery, vol. 26, no. 2, pp. 891-898, 2011.

[5] A. Pittolo and A. M. Tonello, "A synthetic statistical mimo plc channel model applied to an in-home scenario," IEEE Trans. on Commun., vol. 65, no. 6, pp. 2543-2553, 2017.

[6] A. M. Tonello and F. Versolatto, "Bottom-up statistical PLC channel modelingPart II: Inferring the statistics," IEEE Trans. on Power Del., vol. 25, no. 4, pp. 2356-2363, 2010.

[7] A. G. Lazaropoulos, "Statistical Broadband over Power Lines Channel Modeling - Part 1: The Theory of the Statistical Hybrid Model," Progress in Electromagnetics Research C, vol. 92, pp. 1-16, 2019. [Online]. Available: http://www.jpier.org/PIERC/pierc92/01.19012902.pdf

[8] A. G. Lazaropoulos, "Statistical Broadband over Power Lines (BPL) Channel Modeling - Part 2: The Numerical Results of the Statistical Hybrid Model," Progress in Electromagnetics Research C, vol. 92, pp. 17-30, 2019. [Online]. Available: http://www.jpier.org/PIERC/pierc92/02.19012903.pdf

[9] A. G. Lazaropoulos, "Enhancing the Statistical Hybrid Model Performance in Overhead and Underground Medium Voltage Broadband over Power Lines Channels by Adopting Empirical Channel Attenuation Statistical Distribution," Trends in Renewable Energy, vol. 5, no. 2, pp. 181-217, 2019. [Online]. Available: http://futureenergysp.com/index.php/tre/article/view/96/pdf

[10] T. Oliveira, A. Picorone, C. Zeller, S. Netto, and M. Ribeiro, "Statistical Modeling of Brazilian In-Home PLC Channel Features," Journal of Communication and Information Systems, vol. 34, no. 1, pp. 154-168, 2019. 
[11] T. R. Oliveira, C. B. Zeller, S. L. Netto, and M. V. Ribeiro, "Statistical modeling of the average channel gain and delay spread in in-home PLC channels," in Proc. in IEEE International Symposium on Power Line Communications and Its Applications, pp. 184-188, Austin, TX, USA Mar. 2015.

[12] J. A. Cortes, F. J. Canete, L. Diez, and J. L. G. Moreno, "On the statistical properties of indoor power line channels: Measurements and models," in Proc. IEEE International Symposium on Power Line Communications and Its Applications, pp. 271-276, Apr. 2011.

[13] A. M. Tonello, F. Versolatto, and A. Pittolo, "In-home power line communication channel: Statistical characterization," IEEE Trans. on Commun., vol. 62, no. 6, pp. 2096-2106, Jun. 2014.

[14] S. Galli, "A novel approach to the statistical modeling of wireline channels," IEEE Trans. on Commun., vol. 59, no. 5, pp. 1332-1345, May 2011.

[15] A. G. Lazaropoulos and P. G. Cottis, "Transmission characteristics of overhead medium voltage power line communication channels," IEEE Trans. Power Del., vol. 24, no. 3, pp. 1164-1173, Jul. 2009.

[16] A. G. Lazaropoulos and P. G. Cottis, "Broadband transmission via underground medium-voltage power lines-Part I: transmission characteristics," IEEE Trans. Power Del., vol. 25, no. 4, pp. 2414-2424, Oct. 2010.

[17] A. G. Lazaropoulos, "Towards Modal Integration of Overhead and Underground Low-Voltage and Medium-Voltage Power Line Communication Channels in the Smart Grid Landscape: Model Expansion, Broadband Signal Transmission Characteristics, and Statistical Performance Metrics (Invited Paper)," ISRN Signal Processing, vol. 2012, Article ID 121628, pp. 1-17, 2012. [Online]. Available: http://www.hindawi.com/isrn/sp/2012/121628/

[18] A. G. Lazaropoulos, "Virtual Indicative Broadband over Power Lines Topologies for Respective Subclasses by Adjusting Channel Attenuation Statistical Distribution Parameters of Statistical Hybrid Models - Part 2: Numerical Results for the Overhead and Underground Medium-Voltage Power Grids," Trends in Renewable Energy, vol. 5, no. 3, pp. 258-281, Aug. 2019. [Online]. Available: http://futureenergysp.com/index.php/tre/article/view/100/pdf

[19] A. G. Lazaropoulos, "Business Analytics and IT in Smart Grid - Part 2: The Qualitative Mitigation Impact of Piecewise Monotonic Data Approximations on the iSHM Class Map Footprints of Overhead Low-Voltage Broadband over Power Lines Topologies Contaminated by Measurement Differences," Trends in Renewable Energy, vol. 6, no. 2, pp. 187-213, 2020. [Online]. Available: http://futureenergysp.com/index.php/tre/article/download/118/94

[20] A. G. Lazaropoulos, "Statistical Channel Modeling of Overhead Low Voltage Broadband over Power Lines (OV LV BPL) Networks - Part 2: The Numerical Results of Class Map Footprints of Real OV LV BPL Topologies, Branch Line Faults and Hook Style Energy Thefts," Trends in Renewable Energy, vol. 6, no. 1, pp. 88-109, Mar. 2020. [Online]. Available: http://futureenergysp.com/index.php/tre/article/download/113/pdf

[21] I. C. Demetriou and M. J. D. Powell, "Least squares smoothing of univariate data to achieve piecewise monotonicity", IMA Journal of Numerical Analysis, vol. 11, no. 3, pp. 411-432, 1991.

[22] A. G. Lazaropoulos, "Business Analytics and IT in Smart Grid - Part 1: The Impact of Measurement Differences on the iSHM Class Map Footprints of 
Overhead Low-Voltage Broadband over Power Lines Topologies," Trends in Renewable Energy, vol. 6, no. 2, pp. 156-186, 2020. [Online]. Available: http://futureenergysp.com/index.php/tre/article/download/117/93

[23] A. G. Lazaropoulos, "Statistical Channel Modeling of Overhead Low Voltage Broadband over Power Lines (OV LV BPL) Networks - Part 1: The Theory of Class Map Footprints of Real OV LV BPL Topologies, Branch Line Faults and Hook-Style Energy Thefts," Trends in Renewable Energy, vol. 6, no. 1, pp. 61-87, Mar. $2020 . \quad$ [Online]. Available: http://futureenergysp.com/index.php/tre/article/download/112/pdf

[24] A. G. Lazaropoulos, "Virtual Indicative Broadband over Power Lines Topologies for Respective Subclasses by Adjusting Channel Attenuation Statistical Distribution Parameters of Statistical Hybrid Models - Part 1: Theory," Trends in Renewable Energy, vol. 5, no. 3, pp. 237-257, Aug. 2019. [Online]. Available: http://futureenergysp.com/index.php/tre/article/view/99/pdf

[25] A. G. Lazaropoulos, "Improvement of Power Systems Stability by Applying Topology Identification Methodology (TIM) and Fault and Instability Identification Methodology (FIIM)-Study of the Overhead Medium-Voltage Broadband over Power Lines (OV MV BPL) Networks Case," Trends in Renewable Energy, vol. 3, no. 2, pp. 102-128, Apr. 2017. [Online]. Available: http://futureenergysp.com/index.php/tre/article/view/34

[26] A. G. Lazaropoulos, "Detection of Energy Theft in Overhead Low-Voltage Power Grids - The Hook Style Energy Theft in the Smart Grid Era," Trends in Renewable Energy, vol. 5, no. 1, pp. 12-46, Oct. 2018. [Online]. Available: http://futureenergysp.com/index.php/tre/article/view/81/pdf

Article copyright: (C) 2020 Athanasios G. Lazaropoulos. This is an open access article distributed under the terms of the Creative Commons Attribution 4.0 International License, which permits unrestricted use and distribution provided the original author and source are credited. 